\title{
The Chinese Version of WOrk-reLated Flow Inventory (WOLF): An Examination of Reliability and Validity
}

\author{
Yi-yu CHEN ${ }^{1, a}$, Xiao-tong YU, b and Bo HUANG ${ }^{3, c, ~ * ~}$ \\ ${ }^{1}$ Yunan University of Finance and Economics Business School in Kunming city of \\ Yunnan province in China \\ ${ }^{2}$ Yunan University of Finance and Economics Business School in Kunming city of \\ Yunnan province in China \\ ${ }^{3}$ Jingxiuzhuangyuan community 3-1-601, NO.312, Hongshan east road, Kunming city \\ of Yunnan province in China \\ aerdongrain@163.com, byuxiaotong0523@sina.com, 'betty4870@126.com \\ *Bo HUANG
}

Keywords: Reliability, Validity, Work-related flow.

\begin{abstract}
For introducing the WOrk-reLated Flow Inventory which was developed by Bakker (2008) to the research of work-related flow in China, and examing the reliability and validity of the scale, Exploratory and confirmatory factor analysis have showed us that the scale was composed of 3 factors such as, absorption, enjoyment and intrinsic motivation. The Cronbach $\alpha$ of the 3 subscales were between 0.68 and 0.80 , and the Cronbach $\alpha$ of WLOF was 0.87 . Furthermore, the fitting index of model is well. Therefore, the Chinese version of WOLF has showed to be reliable and valid.
\end{abstract}

\section{Introduction}

Recently, pressure for people appears to be growing with the development of social and material living conditions. Therefore it is urgent and important to maintain a positive mental attitude at work. Different disciplines' and fields' scholars studied a host of work-related variables, including positive emotion state as well as flow at work. The notion of flow at work was advocated in the 1970's by Csikszemtmihalyi, who has been studying for the states of optimal experience over 20 years. Flow at work means a state of concentration so focused that it amounts to absolute absorption in an activity. Everyone experiences flow from time to time and will reflect the self-enjoyment emotion state [1]. In early studies, most of them were aimed at special groups of the career, such as musician and professional athlete, and on this basis they developed a series of flow scales, of which Flow State Scale (FSS) and Dispositional Flow Scale (DFS) were the most widely used[2]. Then scholars designed the abbreviated Flow State Scales---FSS-2 and DFS-2. With the further advances of scientific research, the introduction of flow is becoming increasingly important in organizational behavior. In 2008, a new kind of scale called Work-ReLated Flow inventory (WOLF) was developed by Bakker, and this type of scale was specifically designed for the general work fields. However, because of the late arrival, WOLF is seldom used in previous empirical studies in China. Hence, we aim to analyze the WOLF through confirmatory factor analysis and provide foundation for future research. 


\section{Method}

Participants and Procedures. Random sampling was conducted among health care providers, teaching staff and employees of enterprise. The total sample size was 202. The participants were $29.21 \%(\mathrm{~N}=59)$ male and $70.79 \%(\mathrm{~N}=143)$ female. $86.14 \%$ of the participants had master's degree $(\mathrm{N}=174)$. The kurtosis and skewness coefficients were 0.441 and -0.257 respectively, and the absolute values were less than 3 and 10 . These kurtosis's and skewness's levels proved that the sample was normal distribution.

\section{Measures}

The degree to which participants reported having work-related flow experience with the Chinese version of work-related flow inventory (WOLF) which developed by Bakker(2008). The Chinese version of WOLF was translated by experts in organizational behavior and English repeatedly and strictly. The WOLF includes 13 items measuring absorption (4 items), enjoyment (4 items), and intrinsic motivation (5 items). On a five-point Likert scale, item responses ranged from 1 (absolutely disagree) to 4 (absolutely agree).

Quality control. For avoiding the error in survey, two methods were applied in the process of data collection-survey online and field research, and the field of investigation was inclusive. Before the investigation, we tried to mobilize every participant to ensure the authenticity of their answer. Moreover, on the basis of learning this scale, we trained the numbers of investigation team intensively and unified the language of survey. After data collection, we dropped the questionnaires which were completed less than $80 \%$, and re-checked the questionnaires in the cause of logging data at random.

Statistics and analysis tools. To explore the correlation analysis, exploratory factor analysis, and reliability analysis, the software SPASS19.0 and Stata13.0 were used. And, with the software packages of AMOS21.0, it's implemented a confirmatory factor analysis for the 202 sample data.

\section{Results}

Analysis of the items. The 13 items of WOLF and the correlation coefficients for the total scores of this scale are displayed in Table 1 . As can seen from the table, all correlation coefficients were less than $0.3(\mathrm{P}<0.01)$. This means that every item strongly correlated with the general scale.

Table 1.13 items and the correlation coefficients for the total scores of this scale

\begin{tabular}{lcc}
\hline items & $\mathrm{x} \pm \mathrm{s}$ & $\mathrm{r}$ \\
\hline 1. When I am working, I think about nothing else & $3.46 \pm 1.226$ & $0.42^{* *}$ \\
2. I get carried away by my work & $2.58 \pm 1.236$ & $0.75^{* *}$ \\
3. When I am working, I forget everything else around me & $3.09 \pm 1.248$ & $0.61^{* *}$ \\
4. I am totally immersed in my work & $2.93 \pm 1.226$ & $0.67^{* *}$ \\
5. My work gives me a good feeling & $2.74 \pm 1.251$ & $0.79^{* *}$ \\
6. I do my work with a lot of enjoy ment & $2.68 \pm 1.185$ & $0.73^{* *}$ \\
7. I feel happy during my work & $2.74 \pm 1.165$ & $0.77^{* *}$ \\
8. I feel cheerful when I am working & $2.51 \pm 1.156$ & $0.79^{* *}$ \\
9.I would still do this work, even if I received less pay & $2.49 \pm 1.305$ & $0.55^{* *}$ \\
10. I find that I also want to work in my free time & $1.94 \pm 1.181$ & $0.52^{* *}$ \\
11. I work because I enjoy it & $2.16 \pm 1.182$ & $0.77^{* *}$ \\
12. When I am working on something, I am doing it for myself & $3.16 \pm 1.311$ & $0.30^{* *}$ \\
13. I get my motivation from the work itself, and not from the reward for it & $2.35 \pm 1.258$ & $0.65^{* *}$ \\
\hline${ }^{* *}$ :P<0.01 & &
\end{tabular}


Exploratory factor analysis. In order to confirm whether this sample was suitable for factor analysis, we examined KMO and Bartlett's Test of Sphericity. As a rule of thumb, a KMO $>0.7$ indicate that there is lots of repetition in variables. In addition, if the $\mathrm{P}$ value of Bartlett's test is less than 0.05 , we can confirm that the scale has a nice construct validation.

Table 2. KMO and Bart lett's Test of Sphericity

\begin{tabular}{lll}
\hline Index & & Result \\
\hline KMO & & 0.880 \\
& The approximate chi-square value & 1495.365 \\
Bartlett's test & df & 78 \\
& Sig. & 0.00 \\
\hline
\end{tabular}

The value of KMO was 0.88 , and higher than 0.7 , indicated that the partial correlation for the inter-item was strong. Meanwhile, the $\mathrm{P}$ value of Bartlett's test was 0.00 , less than 0.05 . Therefore, the construct of the WOLF was well. An exploratory factor analysis using varimax rotation was carried out on the 13 flow items. 3 eigenvectors were obtained because their eigenvalues was higher than 1 . This analysis resulted in a 3-factors solution, which explained $67.42 \%$ of the variance. As shown in Fig.1, there is a inflection point after the third eigenvector.



Fig. 1 Scree plot of eigenvalues after pca

Reliability. Table 3 displayed the reliability coefficients-- Cronbach $\alpha--$ of three dimensions. As a rule of thumb, a $\alpha$ coefficients higher than 0.07 indicate that the level of reliability is good enough. As showed in the Table3, absorption, enjoyment, and intrinsic motivation correlate moderately high with each other. The $\alpha$ coefficients were acceptable for the three dimensions (from 0.68 to 0.92 ), and satisfactory for the total scale whose Cronbach alpha coefficient was 0.87 .

Table 3. The Cronbach alpha coefficient of scale and each subscales

\begin{tabular}{lll}
\hline Scale and subscales & Items & Cronbach alpha coefficient \\
\hline WOLF & 13 & 0.87 \\
Absorption & 4 & 0.80 \\
Enjoyment & 4 & 0.92 \\
Intrinsic motivation & 5 & 0.68 \\
\hline
\end{tabular}

Confirmatory factor analysis. With the AMOS21.0 computer program, the sample was used to examine whether the three-factor structure of the flow construct could be 
replicated. The maximum likelihood parameter estimation and 200 bootstrap were used for model estimation because parameters estimated are more robust. Generally speaking, it is acceptable for the values of loading were higher than 0.4. As can be seen in Table4, all of the values of factor loading were higher than 0.4 except the forth item of the intrinsic motivation. And the $t$ values ( $C R$ value) of the factor loading were between 23.3 and 40.0, the $\mathrm{P}$ values were all less than 0.01 . These indicated that standardized loading of the scale items on their respective factors were significant.

Table 4. Standardized loading of the scale items

\begin{tabular}{llllll}
\hline & 1 & 2 & 3 & 4 & 5 \\
\hline Absorption & 0.63 & 0.54 & 0.83 & 0.91 & \\
Enjoyment & 0.80 & 0.87 & 0.92 & 0.89 & \\
Intrinsic motivation & 0.48 & 0.53 & 0.87 & 0.16 & 0.59 \\
\hline
\end{tabular}

Meanwhile, we hypothesized single-factor model to compare with the three-factor model. As a rule, a CFI, GFI, and IFI $\geq 0.9$ [3] and RMSEA $\leq 0.08$ indicate a reasonable fit of the model to the data [4]. The fit indices displayed in Table5.

Table 5 The fit indices of work-related flow

\begin{tabular}{lllllllll}
\hline Model & $\chi 2$ & df & $\chi 2 / d f$ & CFI & IFI & RMSEA & GFI & AGFI \\
\hline Single-factor & 439.930 & 65 & 6.768 & 0.743 & 0.745 & 0.169 & 0.722 & 0.611 \\
Three-factor & 196.329 & 62 & 3.167 & 0.908 & 0.909 & 0.104 & 0.872 & 0.812 \\
\hline
\end{tabular}

Note. $\chi 2$, chi-square; df, degrees of freedom; GFI, goodness-of-fit index; AGFI, Adjust Goodness of Fit Index; RMSEA, root mean square error of approximation; CFI, comparative fit index; IFI, incremental fit index

Validity. As displayed in Table5, the three-factor model fit the data significantly better on every fit index than the single-factor model: $\chi 2 / \mathrm{df}<5$; CFI $>0.9$; IFI $>0.9$; GFI $>0.85$; AGFI $>0.8$. It is not high enough for the RMSEA probably because of the small size of the sample.

Furthermore, the content validity of the three flow dimensions incorporated in the WOLF was determined by examining their correlations as shown in Table6. All the intercorrelations coefficients were significant $(\mathrm{P}<0.01)$.

Table6 Pearson Correlation Coefficient

\begin{tabular}{lllll}
\hline & Work-re lated flow & Absorption & Enjoyment & Intrinsic motivation \\
\hline Work-related flow & 0.87 & & & \\
Absorption & $0.7585^{* *}$ & 0.80 & & \\
En joyment & $0.8520^{* *}$ & $0.4043^{* *}$ & 0.72 & \\
Intrinsic motivation & $0.8299^{* *}$ & $0.4417^{* *}$ & $0.6377^{* *}$ & 0.68 \\
\hline
\end{tabular}

Based on the above, and in view of the KMO $(\mathrm{KMO}<0.6)$ and Bartlett's test $(\mathrm{P}<0.01)$, one can draw a conclusion that the three-model of work-related flow had a very good construct validity and content validity.

\section{Discussions}

By investigating 202 samples, our paper tested the reliability and validity of the WOLF in the context of China. Reliability refers to the measurement of scale should be dependable, accurate, stable and consistent. We usually adopt Cronbach $\alpha$ to test reliability and deem that internal consistency is good when Cronbach $\alpha$ is greater than 0.7. In this paper, Cronbach $\alpha$ is greater than 0.7 both in flow sub-scales and the total scale except intrinsic motivation $(\alpha>0.6)$. So we think the WOLF has a good reliability of internal consistency in Chinese context. 
Validity refers to the measurement of scale should be valid and correct. It reflects how well your scale matches characteristics of measuring objects. We tested the construct and content validity of WOLF. Construct validity is the degree to which a test measures what it claims, or purports, to be measuring. First it determined three factors which were completely in conformity with Bakker's assumption in the process of exploratory factors analysis. And then fit index showed a satisfied construct validity in this scale. Finally, we identified the scale also had a good content validity by measuring correlation between total scale and each factor.

Owing to the limitation of objective condition and sample size, the representative of some indicators was non-significant. In the future research, we need to enlarge the sample size and go on with the measurement of WOLF's reliability and validity in the context of China. Moreover, because of the data collection was done at a time, we cannot make a comparison at different time points. Consequently, test-retest reliability and stability of structures are not represented in this paper. Future research can test the WOLF in different time in order to get a more accurate reliability analysis.

\section{References}

[1] Mihalyi Csikszemtmihalyi. Flow: The Psychology of Optimal Experience [J]. Academy of Management Review, 1990, 16(1):75-77

[2] S. A. Jackson, H. W. Marsh. Development and validation of a scale to measure optimal experience: the Flow State Scale.[J]. Journal of Sport \& Exercise Psychology,1996, 18(1) (1):17-35

[3] Rick H. Hoyle. The structural equation modeling approach: Basic concepts and fundamental issues.[J]. R.h.hoyle Structural Equation Modeling Concepts Issues \& Applications .thousand Oaks Ca7 Sage, 1995

[4] M. W. Browne, R. Cudeck. Single Sample Cross-Validation Indices for Covariance Structures[J]. Multivariate Behavioral Research, 1989, 24 (4):445-455 